\title{
Identification of Fluorescent AFLP and SSR Markers for Differentiation and Analysis of New Guinea Impatiens
}

\author{
E.J. Parks and J.W. Moyer ${ }^{1}$ \\ North Carolina State University, Department of Plant Pathology, Raleigh, NC 27695
}

J.H. Lyerly

North Carolina State University, Department of Crop Science. Raleigh, NC 27695

AdDITIONAL INDEX wORDs. molecular differentiation, microsatellite, Impatiens hawkeri, floral crop, DNA fingerprint

\begin{abstract}
AвSTRact. Fluorescent amplified fragment length polymorphism (F-AFLP) and microsatellites (SSRs) were used to evaluate new guinea impatiens (Impatiens hawkeri W. Bull) cultivars. Ninety-five quality-selected polymorphic fragments from $10 \mathrm{~F}-\mathrm{AFLP}+3$ primer combinations were used to evaluate 100 cultivars representing a variety of colors, forms, and breeding programs. Jaccard similarities and unweighted pair-group method of the arithmetic average (UPGMA) clustering formed a dendrogram with three cultivar groups, to a large extent clustering the cultivars by breeder with a high cophenetic correlation coefficient. A small insert genomic library was created and $442 \mathrm{~kb}$ of new guinea impatiens sequence was screened for repetitive motifs, resulting in 14 microsatellite markers. A subset of 46 cultivars representing five commercial breeding companies and 11 cultivar series was selected for microsatellite analysis. Seven loci were polymorphic, with two to six alleles per locus. Although both methods were equally effective in distinguishing the cultivars from one another, the topologies of the dendrograms for the two methods were different. The topology of the AFLP dendrogram reflected possible relationships based on cultivar series and breeding company, while the SSR dendrogram did not. The objectives of this research were to develop and validate both F-AFLP and SSR methodologies for new guinea impatiens, identify markers that can be reliably used for fingerprinting, and create a database for future cultivar comparisons.
\end{abstract}

New guinea impatiens is comprised of a group of wild and hybrid species and cultivars originating from 25 plants collected in a 1970 expedition to New Guinea by the Longwood Foundation and the U.S. Dept. of Agriculture's Agricultural Research Service (USDA-ARS) (Arisumi and Cathey, 1976). This initial collection had a range of characteristics uncommon to the U.S. ornamental market, with large, brightly colored flowers and a variety of leaf shapes and colors. Starting in 1972, the first of many hybrids, including natural and induced tetraploid and amphidiploid lines (Arisumi, 1978), were released to research institutions and commercial and amateur breeders. Today these hybrids, which include those hybridized with species from the islands of Java and Celebes, form the basis for plants in production. Continuous breeding efforts over the past 33 years have dramatically increased the number of commercially available cultivars, which are available in a wide variety of forms, foliage, and flower colors.

New guinea impatiens has become an economically important floral crop, with an annual wholesale value in 2004 of more than $\$ 81$ million in the U.S., placing it in the top 15 species of floriculture crops according to the USDA National Agricultural Statistics Service. Breeders and growers require new tools to monitor adherence to the International Union for the Protection of New Varieties of Plants (UPOV) and U.S. patent law as well as provide much needed data in countries that do not require "distinct, uniform, stable" testing (Ecke, 1998a). Economically

Received for publication 10 Jan. 2006. Accepted for publication 9 May 2006. The authors would like to acknowledge the North Carolina State Arboretum for providing plant material and Dr. Marcia Gumpertz for statistical assistance. This work was supported by Kientzler GmbH \& Co. and the U.S. Dept. of Agriculture Agricultural Research Service (\#58-6645-2-225).

${ }^{1}$ To whom reprint requests should be addressed. Email address: James Moyer@ncsu.edu; Tel.: 919-515-7984; Fax: 919-515-7716 significant intellectual and ethical issues have been raised in the floral crop industry (Ecke, 1998a; Weddington, 2002). Some floriculture growers may fraudulently produce plants, depriving the breeder of royalties (Becher et al., 2000; Ecke, 1998b). As a result, there has been "a significant deterioration of ethics" in the breeding industry (Ecke, 1998a).

Accurate and sensitive cultivar identification is vital to improving this situation. Evaluation of morphological characteristics has been the traditional means of cultivar identification, a difficult and lengthy process (Esselink et al., 2003; Ling et al., 1997). In addition, cultivar differentiation using morphological characteristics has become more difficult due to the increasing phenotypic similarity between cultivars as their numbers have increased (Ecke, 1998a; Esselink et al., 2003). Another obstacle to cultivar identification of new guinea impatiens has been the lack of pedigree data; with many commercial and public institutions involved in breeding this crop from early in its cultivation history, documentation of hybridization and breeding efforts is fragmented. These difficulties not only obscure identification of cultivars, but also may impede selection of appropriate cultivars for comparison for patent purposes.

DNA fingerprinting is a highly effective, precise, efficient tool for cultivar identification, and can be used to assess essentially derived cultivars and detect illegal propagation (Becher et al., 2000; Ecke, 1998b). Methods such as AFLP and SSR, which have been used in a wide variety of crops, are now becoming more commonplace in floral crop analysis. AFLP has been used in many floral crops, including geranium [Pelargonium L'Hér. ex Ait. (Barcaccia et al., 1999)], peruvian lily [Alstromeria L. (Han et al., 1999, 2000)], rose [Rosa L.(Debener et al., 2000)], daylily [Hemerocallis L. (Tomkins et al., 2001)], new guinea impatiens (Carr et al., 2003), and poinsettia [Euphorbia pulcherrima Willd. (Parks and Moyer, 2004)]. Recently, an advancement of AFLP 
methodology known as fluorescent AFLP has been used in seed impatiens [Impatiens walleriana Hook. f. (Carr and Korban, 2004)] and orchid [Dendrobium Ag. (Xiang and Hong, 2003)]. SSR analysis has been used in floral crops such as geranium (Becher et al., 2000) and carnation (Dianthus caryophyllus L.) (Smulders et al., 2003). These molecular tools can provide new guinea impatiens breeders with additional genetic information for breeding purposes, and facilitate cultivar identification to ensure that their rights are protected. Using these methods, a database of genetic fingerprints can be generated, providing a basis for comparison of cultivars and serving as a "silent enforcer" of breeder's rights (Weddington, 2002).

The objectives of this study were: 1) to evaluate the usefulness of F-AFLP to discriminate a large, diverse set of new guinea impatiens cultivars, and determine if F-AFLP could be used to elucidate breeding history, 2) to develop SSR markers for use in new guinea impatiens and determine if this technique could be used for fingerprinting, 3 ) to compare the discriminatory ability and informativeness of the F-AFLP and SSR methods in new guinea impatiens, 4) to create a stable database of genetic markers for future comparison of new guinea impatiens cultivars.

\section{Materials and Methods}

Plant material and DNA isolation. One-hundred cultivars of new guinea impatiens from five major breeding programs were selected for analysis (Table 1). Fully expanded leaf tissue was collected from healthy new guinea impatiens plants maintained in the greenhouse or grown under bedding trial conditions at the North Carolina State Univ. Horticultural Field Laboratory (Raleigh) and stored at $-80^{\circ} \mathrm{C}$. Frozen leaf tissue was ground in liquid nitrogen to a fine powder using a mortar and pestle, and then transferred to a 1.5-mL microcentrifuge tube. DNA was extracted using the Puregene System (Gentra Systems, Minneapolis) with the following modifications. Polyvinylpyrrolidone M.W. 8000 (Acros Organics, Pittsburgh) was added to the Puregene Cell Lysis solution at a concentration of $0.02 \% \mathrm{w} / \mathrm{v}$ to aid in the removal of polysaccharides, and the protein precipitation incubation time was increased to 45-60 min. Samples were resuspended in 50-100 $\mu \mathrm{L}$ "low" TE (10 mm Tris-HCl, pH 8; 0.01 mm EDTA) with 0.3-0.6 $\mu \mathrm{g} \cdot \mu \mathrm{L}^{-1}$ RNase, depending on pellet size, and quantified using agarose gel electrophoresis.

F-AFLP ANALYSIS. AFLP was carried out as described by Vos et al. (1995) using the AFLP Analysis System I (Invitrogen, Frederick, Md.) with the following modifications. Dithiothreitol (Promega, Madison, Wis.) was added to the EcoRI and MseI restriction digest at a concentration of $5.0 \mathrm{~mm}$ to ensure complete digestion. Ligated DNA was used, without dilution, as template for preamplification. Selective amplification was carried out in $10-\mu \mathrm{L}$ reactions using AFLP Amplification Core Mix (Applied Biosystems, Foster City, Calif.), 1:10 diluted preamplified DNA, and primers with three selective nucleotides each. The EcoRI primer was 5' labeled with FAM (MWG Biotech, High Point, N.C.). Electrophoresis was carried out on an ABI Prism 377 DNA sequencer (Applied Biosystems) using 5\% Long Ranger Singel Packs (Cambrex, Walkersville, Md.) and 96-well membrane combs (The Gel Co., San Fransisco). GeneScan-500 ROX standard (Applied Biosystems) was loaded in each lane. F-AFLP fragments were sized using the Local Southern algorithm of GeneScan 2.02 software (Applied Biosystems). Tables were generated from the sizing data and exported to text files. The raw sizing data was binned into size categories, converted to binary data, and formatted using Beagle (Lyerly, 2002). Genographer 1.6.0 software (Benham, 2001) was used to verify scoring. Profiles were validated by comparing duplicated samples for consistency.

Ten primer combinations were identified in a previous study to be most informative for new guinea impatiens based on number of polymorphisms and clarity of banding pattern (Table 2) (J.H. Lyerly, E.J. Parks, and J.W. Moyer, unpublished). In that study, polymorphic fragments amplified by these primers were assigned a quality score based on intensity, resolution, and reproducibility across 43 duplicate samples. Using this method, 95 fragments with quality scores over $80 \%$ were selected in the size range from 85 $\mathrm{bp}$ to $448 \mathrm{bp}$, and these were used in the current study for F-AFLP analysis of 100 cultivars of new guinea impatiens.

Microsatellite isolation AND ANAlysis. New guinea impatiens genomic DNA was partially digested with $0.5 \mathrm{U}$ of $\mathrm{Sau}$ $3 \mathrm{AI}$ (Promega) at $37^{\circ} \mathrm{C}$ for $30 \mathrm{~min}$ in a $50-\mu \mathrm{L}$ volume using 200 ng DNA, $5 \mu \mathrm{g}$ BSA and reaction buffer from the supplier. The reaction was stopped with $3 \mu \mathrm{L}$ of $0.5 \mathrm{~m}$ EDTA ( $\mathrm{pH} 8.0)$. The entire reaction was loaded on a $2 \%$ agarose gel, and fragments $\approx 1000-1200$ bp were excised and extracted using QIAquick Gel Extraction Kit (Qiagen, Valencia, Calif.). The fragments were ligated into the dephosphorylated $\mathrm{Bam} \mathrm{H} 1$ multiple cloning site of the Gem-3Z vector at $4{ }^{\circ} \mathrm{C}$ overnight. Following transformation into JM109 High Efficiency Competent Cells (Promega), colonies were grown on Luria broth/ampicillin (LB/Amp) plates overnight. SP6 and T7 primers were used to screen colonies for correct insert length by transferring a sample of each colony to a $15-\mu \mathrm{L}$ reaction with $1 \mathrm{X}$ PCR buffer, $1.5 \mathrm{~mm} \mathrm{MgCl}_{2}, 200 \mu \mathrm{M}$ dNTPs, $500 \mathrm{~nm}$ each forward and reverse primers, $100 \mathrm{ng}$ BSA, and 0.5 U Taq polymerase (CLP, San Diego) in 96-well PCR plates. Cycling conditions consisted of an initial denaturation of 2 min at $94{ }^{\circ} \mathrm{C}$ followed by 35 cycles of $94{ }^{\circ} \mathrm{C}$ for $30 \mathrm{~s}, 55^{\circ} \mathrm{C}$ for $30 \mathrm{~s}$ and $72{ }^{\circ} \mathrm{C}$ for $1 \mathrm{~min}$. For plasmid DNA isolation, the colonies were inoculated into LB/Amp liquid media in deep-well 96-well plates and grown overnight at $37^{\circ} \mathrm{C}$. Miniprep isolation of plasmid DNA was performed using the R.E.A.L. Prep 96 Plasmid Kit (Qiagen) and the BioRobot 9600 (Qiagen). Sequencing was carried out using a Big Dye terminator cycle sequencing kit and automated sequencing with an ABI 3700 (Applied Biosystems) at the Genome Research Laboratory, North Carolina State Univ. The sequence was scanned for repetitive motifs using SSRIT (Temnykh et al., 2001).

Primers were designed using Primer3 software (Rozen and Skaletzky, 2000) and were synthesized at MWG Biotech. The forward primer for each pair contained an additional 19-bp M13 sequence on the $5^{\prime}$ end for M13 tagged PCR (Rampling et al., 2001). Amplifications were performed in $15-\mu \mathrm{L}$ reactions containing: 1X PCR buffer, $1.5 \mathrm{~mm} \mathrm{MgCl}_{2}, 200 \mu \mathrm{M}$ dNTPs, $0.5 \mathrm{pm}$ each unlabeled primer, 1 pM M13 (-29) IRD 700-labeled primer, 20 ng DNA, 1.2 U Taq polymerase (CLP). Cycling conditions consisted of: initial denaturation at $95{ }^{\circ} \mathrm{C}$ for $4 \mathrm{~min}$; 15 cycles of $94^{\circ} \mathrm{C}$ for $30 \mathrm{~s}, 65^{\circ} \mathrm{C}$ for $30 \mathrm{~s}\left(-1{ }^{\circ} \mathrm{C} /\right.$ cycle $), 72^{\circ} \mathrm{C}$ for $80 \mathrm{~s}$; 30 cycles of $94{ }^{\circ} \mathrm{C}$ for $15 \mathrm{~s}, 50^{\circ} \mathrm{C}$ for $15 \mathrm{~s}, 72{ }^{\circ} \mathrm{C}$ for $45 \mathrm{~s}$; and a final extension of $72^{\circ} \mathrm{C}$ for $10 \mathrm{~min}$. BSA was used with primer pair 8-22 at a reaction concentration of $0.5 \mu \mathrm{g} \cdot \mu \mathrm{L}-1$ to increase amplification products to acceptable levels. Amplified products were electrophoresed using LI-COR Fragment Analysis System using 6.5\% KB ${ }^{\text {Plus }}$ Gel Matrix (LI-COR Biosciences, Lincoln, Nebr.). Fragment sizes were determined based on comparison to the LI-COR 50-350 size standard using ProRF2 software (DNA ProScan, Nashville), and the resulting binary data was exported to Microsoft Excel (Microsoft Corp., Redmond, Wash.). 
Table 1. New guinea impatiens cultivars used for fluorescent AFLP and SSR analysis. Code indicates labeling of cultivars in dendrograms.

\begin{tabular}{|c|c|c|c|c|c|c|c|}
\hline Breeder $^{2}$ & Series & Cultivar & Code & Breeder & Series & Cultivar & Code \\
\hline Ball & Celebration & $\begin{array}{l}\text { Bonfire Orange } \\
\text { Improved }\end{array}$ & CBonOrImp ${ }^{y}$ & Dümmen & Petticoat & Red Star & PRdStar \\
\hline Ball & Celebration & Cherry Red & CChRed & Dümmen & Petticoat & Red & PRed $^{y}$ \\
\hline Ball & Celebration & Neon Salmon & $\mathrm{CNeSal}$ & Dümmen & Petticoat & Salmon & PSalmon \\
\hline Ball & Celebration & Raspberry Rose & CRaRose $^{\mathrm{y}}$ & Dümmen & Petticoat & White & PWhite \\
\hline Ball & Celebration & Red Improved & CRedImp $^{y}$ & Dümmen & Riviera & Alegro & RAlegro \\
\hline Ball & Celebration & Sunset Apricot & CSunApr $^{\mathrm{y}}$ & Dümmen & Riviera & Blue Night & RBINight $^{\mathrm{y}}$ \\
\hline Ball & Celebration & White & CWhite $^{\mathrm{y}}$ & Dümmen & Riviera & Blue Star & RB1Star \\
\hline Ball & Celebrette & Hot RoseImproved & CtHtRseImp ${ }^{y}$ & Dümmen & Riviera & Blue & RBlue \\
\hline Ball & Celebrette & Light Lavender & $\mathrm{CtLtLav}^{\mathrm{y}}$ & Dümmen & Riviera & Deep Salmon & RDpSal \\
\hline Ball & Celebrette & Purple & CtPurple ${ }^{y}$ & Dümmen & Riviera & Fire & RFire \\
\hline Ball & Celebrette & Red & $\mathrm{CtRed}^{\mathrm{y}}$ & Dümmen & Riviera & Hot Pink & RHotPk \\
\hline Ball & Celebrette & Strawberry Star & CtStStar & Dümmen & Riviera & Neon & RNeon \\
\hline Ball & Java & Cherry Rose & $\mathrm{JvChRose}^{\mathrm{y}}$ & Dümmen & Riviera & Pink & RPink \\
\hline Ball & Java & Orange & JvOrange $^{\mathrm{y}}$ & Dümmen & Riviera & Red Star & RRdStar \\
\hline Ball & Java & Pearl & JvPearl $^{\mathrm{y}}$ & Dümmen & Riviera & Red & RRed \\
\hline Danzinger & Harmony & Cherry Rose & HChRose $^{y}$ & Dümmen & Riviera & White Eye & RWhEye \\
\hline Danzinger & Harmony & Conga & HConga & Dümmen & Riviera & White & RWhite \\
\hline Danzinger & Harmony & Dark Red & HDkRed & Dümmen & Sweetie & Blue & SwBlue \\
\hline Danzinger & Harmony & Deep Salmon & HDpSal & Dümmen & Sweetie & Cherry & SwCherry \\
\hline Danzinger & Harmony & Flame & HFlame & Dümmen & Sweetie & Fire & SwFire \\
\hline Danzinger & Harmony & Grape & HGrape ${ }^{y}$ & Dümmen & Sweetie & Lavender & SwLav \\
\hline Danzinger & Harmony & Lavender Purple & HLavPur & Dümmen & Sweetie & Pink & SwPink $^{y}$ \\
\hline Danzinger & Harmony & Light Coral & HLtCoral $^{\mathrm{y}}$ & Dümmen & Sweetie & Purple & SwPurple \\
\hline Danzinger & Harmony & Light Pink & HLtPink & Dümmen & Sweetie & Red Star & SwRdStar \\
\hline Danzinger & Harmony & Light Purple & HLtPurple $^{\mathrm{y}}$ & Fisher & Super Sonic & Burgundy & SSBurg $^{\mathrm{y}}$ \\
\hline Danzinger & Harmony & Magenta & HMagenta $^{y}$ & Fisher & Super Sonic & CherryCream & SSChCream \\
\hline Danzinger & Harmony & Orange & HOrange & Fisher & Super Sonic & Coral Ice & SSCorIce \\
\hline Danzinger & Harmony & Peach & HPeach $^{y}$ & Fisher & Super Sonic & Flame & SSFlame $\mathrm{y}^{\mathrm{y}}$ \\
\hline Danzinger & Harmony & Pink & HPink $^{y}$ & Fisher & Super Sonic & Hot Pink & SSHtPink $^{y}$ \\
\hline Danzinger & Harmony & Purple Eye & HPurEye & Fisher & Super Sonic & Lavender & SSLav $^{y}$ \\
\hline Danzinger & Harmony & Purple & HPurple & Fisher & Super Sonic & Magenta & SSMagen \\
\hline Danzinger & Harmony & Royal Red & HRoyRed $^{\mathrm{y}}$ & Fisher & Super Sonic & Orchid & SSOrchid $^{\mathrm{y}}$ \\
\hline Danzinger & Harmony & Salmon & HSalmon & Fisher & Super Sonic & Pastel & SSPaPink \\
\hline Danzinger & Harmony & Super & HSupRed & Fisher & Super Sonic & Peach & SSPeach \\
\hline Danzinger & Harmony & Tangerine & HTang $^{\mathrm{y}}$ & Fisher & Super Sonic & Red & $\operatorname{SSRed}^{\mathrm{y}}$ \\
\hline Danzinger & Harmony & Violet & HViolet $^{y}$ & Fisher & Super Sonic & Salmon & $\mathrm{SSSal}^{\mathrm{y}}$ \\
\hline Danzinger & Harmony & White & HWhite $^{y}$ & Fisher & Super Sonic & Scarlet & SSScarlet $^{y}$ \\
\hline Dümmen & Petticoat & Blue Star & PB1Star & Fisher & Super Sonic & White & SSWhite $^{\mathrm{y}}$ \\
\hline Dümmen & Petticoat & Blue Night & PBlue & Kientzler & Niño & Juan & Juan \\
\hline Dümmen & Petticoat & Fire & PFire & Kientzler & Paradise & Bright Red & PaBrRed ${ }^{y}$ \\
\hline Dümmen & Petticoat & Gamba & PGamba & Kientzler & Paradise & Light Pink & PaLtPink $^{y}$ \\
\hline Dümmen & Petticoat & Hot Rose & PHtRose ${ }^{y}$ & Kientzler & Paradise & Orange & PaOrange $^{\mathrm{y}}$ \\
\hline Dümmen & Petticoat & Lavender & PLav & Kientzler & Paradise & Salmon & PaSalmon $^{\mathrm{y}}$ \\
\hline Dümmen & Petticoat & Mandarin & PMand & Kientzler & Paradise & True Red & PaTrRed ${ }^{y}$ \\
\hline Dümmen & Petticoat & Neon Night & PNeNight $^{y}$ & Kientzler & Paradise & Timor & Timor \\
\hline Dümmen & Petticoat & Orange & POrange & Kientzler & Pizzazz & Purple & PzPurple ${ }^{y}$ \\
\hline Dümmen & Petticoat & Orange Star & POrStar & Kientzler & Pizzazz & Salmon & PzSalmon $^{\mathrm{y}}$ \\
\hline Dümmen & Petticoat & Pink Charme & PPkChar & Kientzler & Pure Beauty & Impr. Delias & ImpDeliass $^{y}$ \\
\hline Dümmen & Petticoat & Pink Eye & PPkEye & Kientzler & Pure Beauty & Marpesia & Marpesia \\
\hline Dümmen & Petticoat & Pink Night & PPkNight $^{\mathrm{y}}$ & Kientzler & Pure Beauty & Red on Pink & PBRdonPk \\
\hline
\end{tabular}

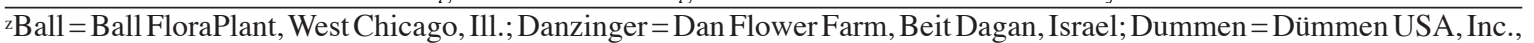
Hilliard, Ohio; Fisher = Fisher USA, Inc., Boulder, Colo.; Kientzler = Kientzler Jungpflanzen, Gensingen, Germany.

yCultivars selected for microsatellite analysis.

AlLele SEQuencing. Samples displaying a nonstepwise pattern of alleles were electrophoresed on the LI-COR Fragment Analysis System using an $8 \%$ denaturing acrylamide gel and a 64-well comb with teeth removed to create triple-width bands. The separation was allowed to continue until the largest fragments of interest were visible on the gel image. The plates were separated and the back plate, with the gel, was reversed onto the scanning surface of the LI-COR Odyssey System (LI-COR Biosciences). A grid was drawn on the plate to aid in fragment localization. The gel was scanned, and the fragments were located and excised. The gel pieces were incubated in $1 \mathrm{X} \mathrm{TE}$ for $20 \mathrm{~min}$ at $95^{\circ} \mathrm{C}$ and 1 $\mu \mathrm{L}$ was used to reamplify the fragment with the locus-specific primers using the SSR PCR conditions. The fragments were then sequenced using $5 \mu \mathrm{L}$ of the reamplified products in the Big Dye terminator cycle sequencing kit and automated sequencing with an ABI 3700 (Applied Biosystems) at the Genome Research Laboratory, North Carolina State Univ., Raleigh.

Statistical analysis. Binary F-AFLP and SSR data were imported into NTSys PC 2.0 (Exter Software, Setauket, N.Y.) for genetic analysis. Jaccard similarity coefficients (Jaccard, 1908) were calculated and clustering was generated using the UPGMA method. Principle coordinates analysis was performed using the DCenter and Eigen programs of NTSys (Exter Software). Cophenetic correlation coefficient was calculated for dendrograms and principle coordinates diagrams using the Coph and MxComp programs of NTSys. Heterozygosity was calculated 
Table 2. Fluorescent AFLP primers selected for cultivar identification analysis of new guinea impatiens cultivars. $\mathrm{E}=$ GACTGCGTACCAATTC and $\mathrm{M}=$ GATGAGTCCTGAGTAA. Selected polymorphic fragments are the subset of polymorphic fragments with quality scores over $80 \%$ used for cultivar analysis.

\begin{tabular}{lccc}
\hline AFLP & $\begin{array}{c}\text { Putative } \\
\text { polymorphic } \\
\text { fragments } \\
\text { primer pair }\end{array}$ & $\begin{array}{c}\text { Selected } \\
\text { polymorphic } \\
\text { fragments } \\
\text { (no.) }\end{array}$ & $\begin{array}{c}\text { Size range of } \\
\text { scored } \\
\text { polymorphisms } \\
\text { (bp) }\end{array}$ \\
\hline E-AAG/M-CAA & 70 & 6 & $133-361$ \\
E-AAG/M-CAG & 88 & 13 & $85-347$ \\
E-AAG/M-CTA & 89 & 4 & $121-222$ \\
E-AAG/M-CTC & 79 & 8 & $109-369$ \\
E-AAG/M-CTG & 78 & 12 & $86-446$ \\
E-ACA/M-CAA & 72 & 7 & $94-448$ \\
E-ACT/M-CAA & 78 & 16 & $92-440$ \\
E-ACT/M-CAG & 51 & 4 & $92-221$ \\
E-ACT/M-CTC & 49 & 14 & $99-427$ \\
E-ACT/M-CTT & 82 & 11 & $91-321$ \\
Total & 736 & 95 & \\
\hline
\end{tabular}

as a comparative measure of information content, using Excel (Microsoft Corp.), for each AFLP and SSR primer pair with the formula $H_{e p}=1-\Sigma p_{i}^{2}$ where $p_{i}$ is the allele frequency for the $i$ th allele (Belaj et al., 2003).

An analysis of molecular variance (AMOVA) was carried out using WINAMOVA 1.04 (Excoffier et al., 1992) on a matrix of squared Euclidean distances calculated using NTsys (Exeter Software). Variance components for the marker systems were calculated with WINAMOVA at each hierarchical level: among cultivars within series, among series within breeder, and among breeder. The degree of differentiation was summarized as proportion of total variance and as $\Phi$ statistics. Significance testing was conducted using WINAMOVA to calculate the null distribution of the $\Phi$ values using a nonparametric permutation, and the $P$ values were calculated after 1000 permutations.

\section{Results}

F-AFLP. F-AFLP was able to fully differentiate all 100 cultivars of new guinea impatiens evaluated in this study. Jaccard similarities ranged from 0.819 between 'Harmony White' and 'Harmony Violet' to 0.242 between 'Celebrette Light Lavender' and 'Java Pearl' and also between 'Super Sonic White' and 'Celebration Raspberry Rose'. The average Jaccard similarity was 0.481 . Heterozygosity values for each primer pair ranged from 0.68 to 0.93 , with an average over primer pairs of 0.84 .

UPGMA clustering generated three major clades (Fig. 1). The first clade included 66 cultivars from four breeding companies; all 22 of Danzinger's Harmony series cultivars, Dummen's 17 Petticoat, seven Sweetie, and 13 Riviera cultivars, three of Fisher's Super Sonic cultivars, one each of Kientzler's Paradise and Niño cultivars, and two of Kientzler's Pure Beauty cultivars. The second clade included 20 cultivars; all of Ball FloraPlant's seven Celebration and five Celebrette cultivars, Kientzler's two Pizzazz, and Kientzler's remaining one Pure Beauty and five Paradise cultivars. Fisher's 11 Super Sonic series and Ball FloraPlant's three Java cultivars comprised the third clade of 14 cultivars. The cophenetic correlation coefficient for the dendrogram was 0.810 .

AMOVAanalysis revealed differentiation between breeder and series. The majority of the variance was among cultivars within series $(77.83 \%)$, but an appreciable amount was among series within breeder $(10.75 \%)$ and among breeders $(11.42 \%)$. Breed- ers were significantly differentiated $\left(\Phi_{\mathrm{CT}}=0.114, P<0.001\right)$, as were series $\left(\Phi_{\mathrm{SC}}=0.121, P<0.001\right)$.

SSR.Approximately 442,000 bases of genomic sequence from 724 clones were identified for new guinea impatiens. The sequence was searched for the presence of SSR motifs of significant size to be polymorphic based on previous studies (Alvarez et al., 2001; Cardle et al., 2000). A total of 18 microsatellite motifs were obtained. An equal number of dinucleotide and trinucleotide motifs were found; each of these classes had six motifs. One each of tetranucleotide, hexanucleotide, and heptanucleotide class was found. Finally, three compound repeats were found, two perfect, and one imperfect, interrupted by a single nucleotide.

Primers were designed for 14 of the primer pairs (Table 3); primers could not be designed for four of the motifs. Two of the motifs were too near the end of the genomic fragment to design a flanking primer. One motif was a duplicated sequence. Finally, one of the motifs was very close to another, so primers were designed flanking the two together.

Forty-six of the new guinea impatiens cultivars were selected for the microsatellite analysis. Cultivars were selected to represent all clusters of the F-AFLP dendrogram, with the full range of Jaccard similarities. For this set of cultivars, seven of the primer pairs generated polymorphic alleles and five yielded only one monomorphic allele. One primer pair did not amplify, and one amplified a pattern too complex to reliably score. The number of alleles each polymorphic primer pair generated ranged from two to six, with a mean of 3.7 alleles. Each cultivar possessed between one and four alleles per primer pair.

Primer pair 8-22 produced five fragments with a size difference of up to $76 \mathrm{bp}$. The predicted amplicon size based on the original sequence was $206 \mathrm{bp}$. Amplification with this primer pair resulted in fragments of 205, 206, 222, 224, and $281 \mathrm{bp}$. To determine which fragments should be scored as alleles, the sequences of the different fragments were compared. Alignment of the fragments with the original cloned genomic sequence for this locus revealed that all of the sequences were related, and that nonstepwise mutations had occurred. The $1 \mathrm{bp}$ difference between the lowest set of alleles, 205 and 206 bp, was due to a single base insertion at position 130, 22 bases upstream from the repetitive region. The middle set of alleles had an $18 \mathrm{bp}$ insertion starting at position 116 , ending $37 \mathrm{bp}$ from the repetitive region, along with the same single base insertion found in the 206 allele. Finally, the large allele had the same insertion as the middle and lower alleles, along with several other insertions, but still maintained the same repetitive region and primer binding sites. All fragments were scored as alleles.

The microsatellite analysis was able to differentiate all 46 cultivars of new guinea impatiens. Jaccard similarities for the SSR data ranged from 0.933 between 'Paradise Orange' and 'Super Sonic Flame' to 0.304 between 'Celebrette Hot Rose Improved' and 'Java Orange', with an average similarity of 0.612 . The cophenetic correlation coefficient for this dendrogram was 0.575. Heterozygosity for the SSRs ranged from 0.49 to 0.78 , with an average value of 0.64. AMOVA analysis of the microsatellite distances partitioned the majority of the variance among cultivars within series (95.64\%), with a small amount among series within breeder $(1.65 \%)$ and among breeders $(2.71 \%)$. Differentiation was not significant $\left(\Phi_{\mathrm{CT}}=0.027, P>0.05 ; \Phi_{\mathrm{SC}}=0.017, P>0.05\right)$.

COMPARISON OF MARKER METHODS. For the comparative F-AFLP and SSR analysis, a dendrogram was first constructed using the F-AFLP data from the smaller set of 46 cultivars to determine if the clustering as seen in the larger F-AFLP dendrogram was 
Table 3. Characteristics of the SSR loci identified in 442,000 bases of new guinea impatiens DNA sequence.

\begin{tabular}{|c|c|c|c|c|c|}
\hline Locus & Motif & $\begin{array}{l}\text { Alleles } \\
(\text { no. })^{z}\end{array}$ & $\mathrm{PM}^{\mathrm{y}}$ & $\begin{array}{l}\text { Primers }\left(5^{\prime}-3^{\prime}\right)^{\mathrm{x}} \\
\text { Forward; Reverse }\end{array}$ & $\begin{array}{l}\text { Size } \\
(\mathrm{bp})^{\mathrm{W}}\end{array}$ \\
\hline \multirow[t]{2}{*}{$8-22$} & $(\mathrm{ag})_{7}$ & 5 & $\mathrm{PM}$ & CGGTCAACAAGAGAGGAAGC & 206 \\
\hline & & & & TTTCGATCTCCTGGCTATGG & \\
\hline \multirow[t]{2}{*}{$3-38$} & $(\mathrm{tc})_{10}$ & 4 & PM & GGCAGTGAGGTGATTTCCAT & 236 \\
\hline & & & & CGCTCGCTCATTCCATATTC & \\
\hline \multirow[t]{2}{*}{$13-77$ (a) } & $(\mathrm{ct})_{7}$ & 4 & PM & CGATTTTCATCTATTCCGGTTT & 154 \\
\hline & & & & TTAGCCGTCTTGACCTTTCC & \\
\hline \multirow[t]{2}{*}{$3-27$} & $(\operatorname{tga})_{5}$ & 4 & PM & TACCGGTGGTGGTTATGAGG & 151 \\
\hline & & & & GTAAACATCGGGGGTCACAT & \\
\hline \multirow[t]{2}{*}{$7-21$} & $(\text { at })_{9}$ & 3 & PM & CCGCTCACTATGGACGAGAT & 161 \\
\hline & & & & CCTCACAGGCCGTTTACTTC & \\
\hline \multirow[t]{2}{*}{$5-58$} & $(\text { gat })_{6}$ & 3 & PM & TGGAAAGGGTGGATGATAGC & 236 \\
\hline & & & & ATTGATCCTGCGTGGATGTT & \\
\hline \multirow[t]{2}{*}{$11-93$} & $(\mathrm{tc})_{6}$ & 2 & PM & ACAAAAACAAGCAGCGAACA & 171 \\
\hline & & & & CTAGGCTTCTATTTCACTCAACATC & \\
\hline \multirow[t]{2}{*}{$27-78$} & $(\mathrm{cct})_{5}$ & 1 & NP & AAGGAATCCAATCCAGCTCA & 210 \\
\hline & & & & GGGAGCGAAGTGTTGAAGAG & \\
\hline \multirow[t]{2}{*}{$7-82$} & $(\text { attcga })_{3}$ & 1 & NP & ACССТССАСАТССССАТАТT & 195 \\
\hline & & & & CGTGAGAATTTCGTGGTGAA & \\
\hline \multirow[t]{2}{*}{$27-40(a)$} & $(\text { tat })_{10}$ & 1 & NP & CATGATTTCATAGAATTTTTGATTCG & 300 \\
\hline & & & & GGATCTGACGAACGCTCTCT & \\
\hline $27-40(b)$ & $(\text { aaaataa })_{2}$ & 1 & NP & amplified with 27-40 (a) & - \\
\hline \multirow[t]{2}{*}{$27-40(\mathrm{c})$} & $(\mathrm{at})_{10}$ & 1 & NP & TTCAGGGGCCAAGTACAATC & 229 \\
\hline & & & & TTTGATTTAAAGGGGGAATCA & \\
\hline \multirow[t]{2}{*}{$28-63$} & $(\operatorname{agc})_{4}(\operatorname{tg})_{3}$ & 1 & NP & GCCTTCAAAGTTGCTTCTGC & 244 \\
\hline & & & & TCTTGGCATCCCCATTGTAT & \\
\hline \multirow[t]{2}{*}{$7-85$} & $(\operatorname{agc})_{3}(\mathrm{cgc})_{3}$ & - & n.d. & CGGACTTCCTTGATGACGTT & 209 \\
\hline & & & & GTTGACCAGCTGAGCGAACT & \\
\hline \multirow[t]{2}{*}{$12-34$} & $(\operatorname{cgg})_{3}$ & - & n.d. & GATGTTGAACTGGACGTTGC & 231 \\
\hline & $\mathrm{c}(\mathrm{gct})_{3}$ & & & GGACGAAGTTTGGAACAAGG & \\
\hline
\end{tabular}

${ }^{\mathrm{z}}$ Based on 46 new guinea impatiens cultivars.

yPolymorphism: $\mathrm{PM}=$ polymorphic; $\mathrm{NP}$ = not polymorphic; n.d. = not determined, locus would not amplify.

${ }^{x}$ For M13 tagged PCR, the sequence (5'-3') CACGACGTTGTAAAACGAC was added to the $5^{\prime}$ end of the forward primer.

${ }^{w}$ Size of sequenced allele. Size does not include 19 bases from M13-tagged PCR.

maintained. Only two changes were observed; 'Riviera Blue Night' changed from an outgroup of cluster 1 in the large dendrogram to an outgroup of clusters 1 and 2 in the small dendrogram, and 'Celebration Sunset Apricot' changed from an outgroup of cluster 2 in the large dendrogram to an outgroup of clusters 1 and 2 in the small dendrogram. The dendrogram resulting from the microsatellite data maintains very little of the clustering of the F-AFLP dendrogram (Fig. 2). There were no obvious clusters of cultivars. Generally, the cultivars comprising the clusters of the F-AFLP dendrogram were scattered throughout the SSR dendrogram.

Principle coordinates analysis of the F-AFLP data was consistent with the clustering of the F-AFLPdendrogram(Fig. 3a). Three distinct clusters are visible, corresponding to the three clusters in the F-AFLP dendrogram, with a cophenetic correlation coefficient of 0.758 . In contrast, the PCA of the microsatellite data does not correspond to the SSR dendrogram (Fig. 3b). Approximately two-thirds of the cultivars clustered together in the lower portion of the graph, and the remaining third was in the upper portion, with a distinct division between the two groups. In addition, some series clustering can be seen. There are three clusters that contain nine of the 11 Harmony cultivars. Two clusters contain seven of nine Super Sonic cultivars. Finally, one cluster contains two of four Celebrette cultivars. The cophenetic correlation coefficient for the SSR PCA was 0.723 .

\section{Discussion}

Very few molecular studies have been conducted in new guinea impatiens (Carr et al., 2003); however the increasing popularity of new guinea impatiens in the ornamental market has brought about the need for new tools. This study presents the first reported use of fluorescent AFLP in new guinea impatiens. One hundred cultivars from five major breeding programs were differentiated, demonstrating the value of this technique for distinguishing cultivars, even those that are similar morphologically.

Fluorescent methods present several advantages over AFLP in which radioactively labeled primers are used (Carr and Korban, 2004). It increases scoring accuracy by eliminating uncertainty in fragment sizing and intensity (De Riek et al., 1999). Fragment presence and absence is based not on visual inspection, but on a numerical value of fluorescent signal intensity, which can be used to assign a minimum acceptable level for a faint band. As well as using an internal lane standard in every lane, GeneScan software can also be used to perform sizing comparisons using 


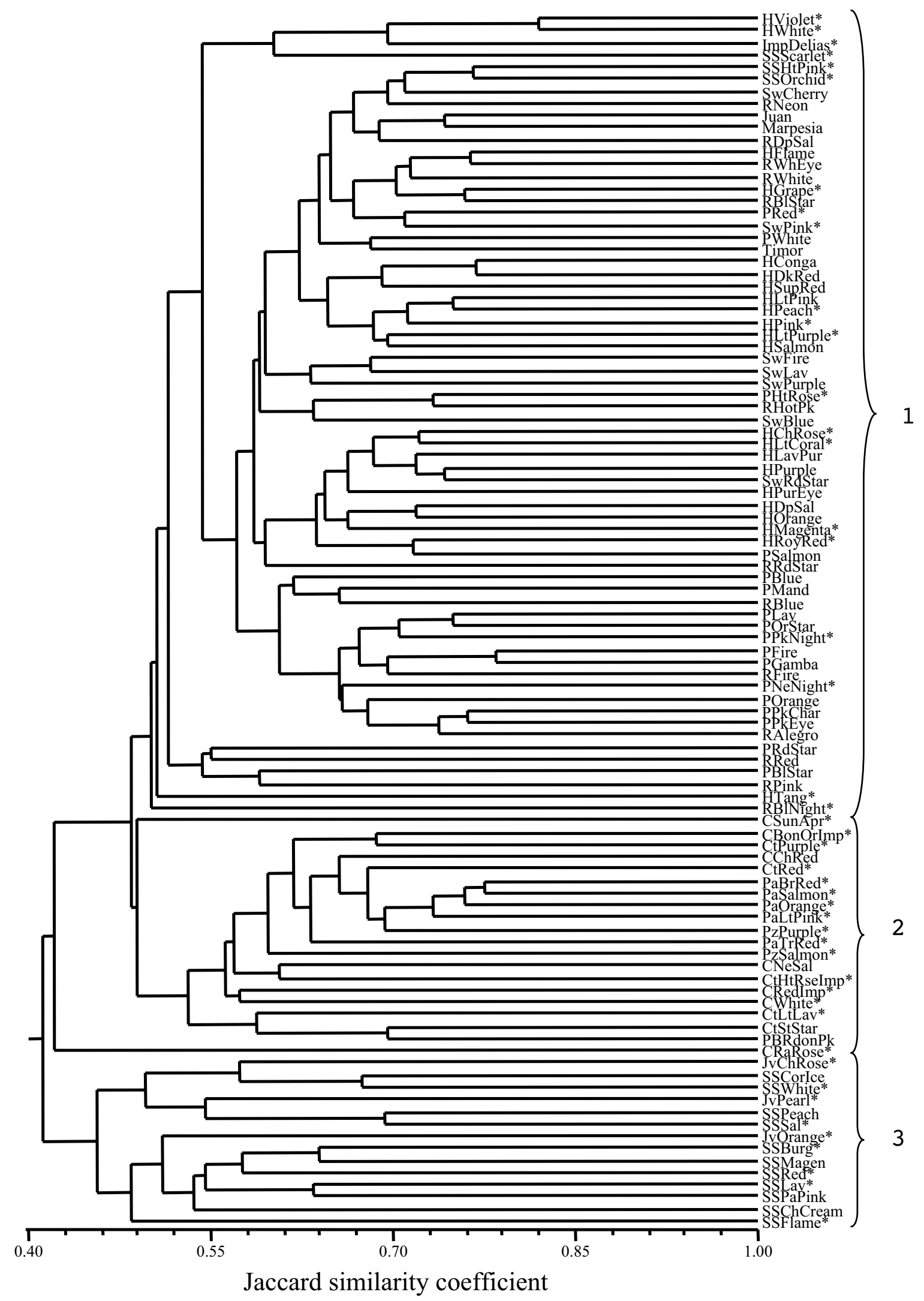

Fig. 1. Dendrogram of 100 new guinea impatiens cultivars generated using Jaccard's similarity coefficient and UPGMA clustering on F-AFLP data. Three major clusters are indicated. Marked cultivars $\left(^{*}\right)$ are those chosen for further analysis with microsatellites. Abbreviations are defined in Table 1. 


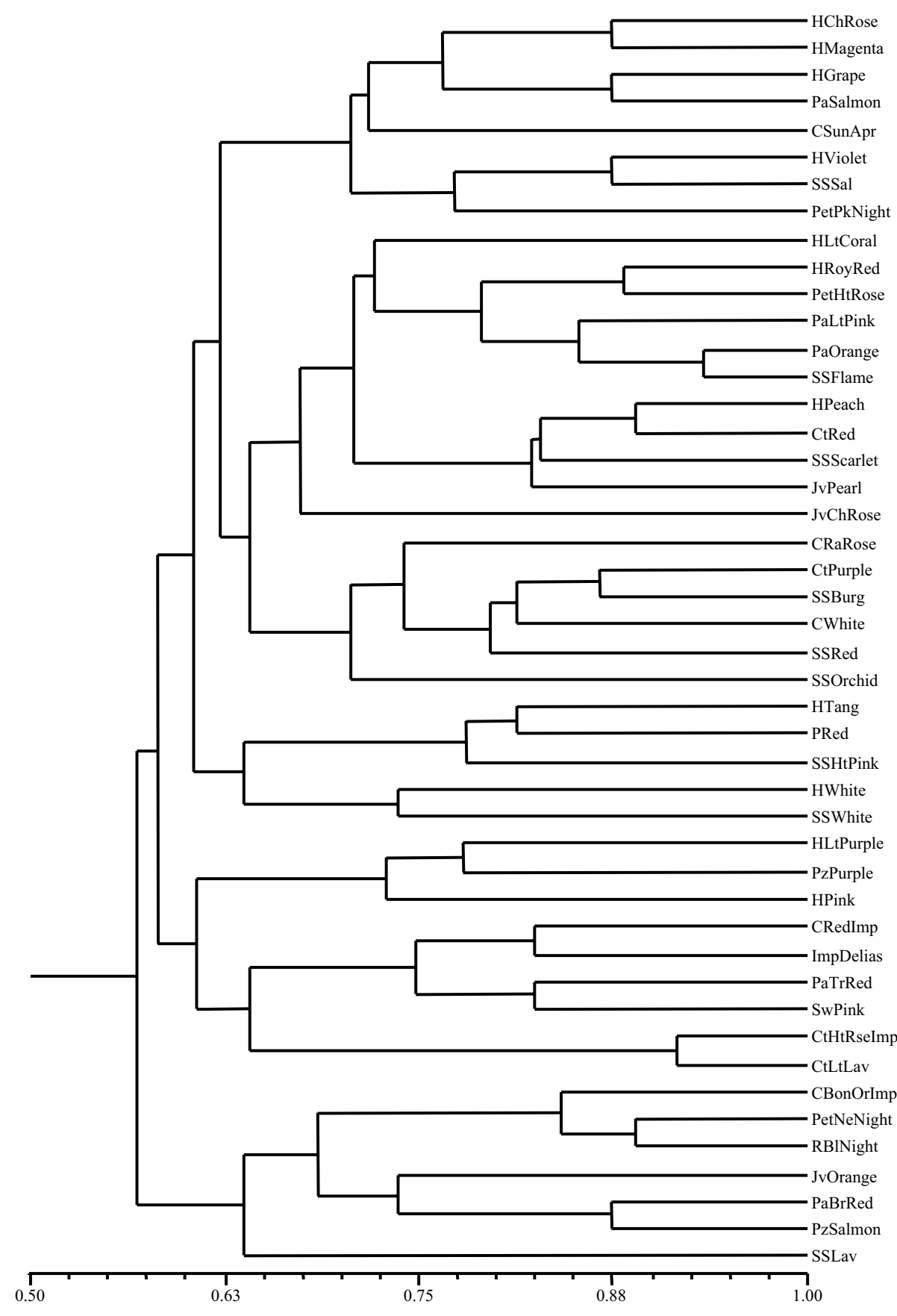

Jaccard Similarity Coefficient

Fig. 2. Dendrogram of 46 new guinea impatiens cultivars using Jaccard similarity coefficient and UPGMA clustering on microsatellite data. Abbreviations are defined in Table 1.

an overlay of sample banding patterns, increasing the accuracy of scoring. In addition, conversion to a fluorescent technique paves the way for more high-throughput analysis since this technique allows greater flexibility with multiplexing.

Cultivars in this study were successfully discriminated from one another using polymorphisms objectively selected based on quality scores. This differs from studies in which all polymorphisms from all tested primers are utilized for determining relationships (Han et al., 2000; Incirli and Akkaya, 2001), or where primers are selected randomly based on visual inspection, and the polymorphisms generated by specific combinations sub- sequently scored (Carr et al., 2003). The importance of consistent polymorphism selection and validation was demonstrated in azalea (Rhododendron simsii Planch.) (De Riek et al., 1999), which showed that analysis with validated markers show high correlation with known pedigrees. Even with the purported reproducibility of AFLP, experimental replication is important to avoid false polymorphisms (Debener et al., 2000; Parks and Moyer, 2004).

This study is also the first report of microsatellites in new guinea impatiens. The data in this study estimates the frequency of SSRs motifs in new guinea impatiens to be about $1 / 26 \mathrm{~kb}$, significantly less the $1 / 6-7 \mathrm{~kb}$ estimated for barley (Hordeum vulgare $\mathrm{L}$.), rice (Oryza sativa L.), tomato (Lycopersicon esculentum Mill.), potato (Solanum tuberosum L.), and Arabidopsis thaliana (L.) Heynh. (Cardle et al., 2000), but more than the $1 / 35 \mathrm{~kb}$ in poinsettia (E.J. Parks and J.W. Moyer, unpublished). The proportion of amplifiable primer pairs and the degree of polymorphism obtained was similar to other studies of floral crops. The percentage of primers with clear amplification was high, $85.7 \%$, compared to studies of carnation $[72.7 \%$ (Smulders et al., 2003)] and Pelargonium [56.1\% (Becher et al., 2000)], likely due to the amplification methodology used in the current study. The proportion of polymorphic primers, $50 \%$, was lower than these crops; all eight of the amplified carnation primer pairs yielded polymorphism (Smulders et al., 2003), and 28 of $32(87.5 \%)$ of the Pelargonium primer pairs were polymorphic (Becher et al., 2000). This may be attributed to the narrow genetic base of this crop. The number of amplified alleles for individual loci reflected the tetraploid nature of the species. Even with the lower level of polymorphism, microsatellites were able to differentiate a set of 46 cultivars over a range of similarities.

The nonstepwise mutation pattern that was seen with one microsatellite primer, $8-22$, was investigated with sequence analysis. This revealed that three potentially related loci were amplified by this primer pair. The sequence of these was consistent with duplication events followed with divergence by insertion events. In addition, two of these loci also have alleles that share a single base pair deletion in the flanking region. These types of variation in microsatellites have been described in many crops, including olive (Olea europaea L.) (Carriero et al., 2002) and Pelargonium (Becher et al., 2000).

While F-AFLPs and SSRs were equally able to differentiate all of the new guinea impatiens cultivars tested, they resulted in very different clustering patterns. In the F-AFLPanalysis, cultivars clustered by breeder and series in the dendrogram. The AMOVA 

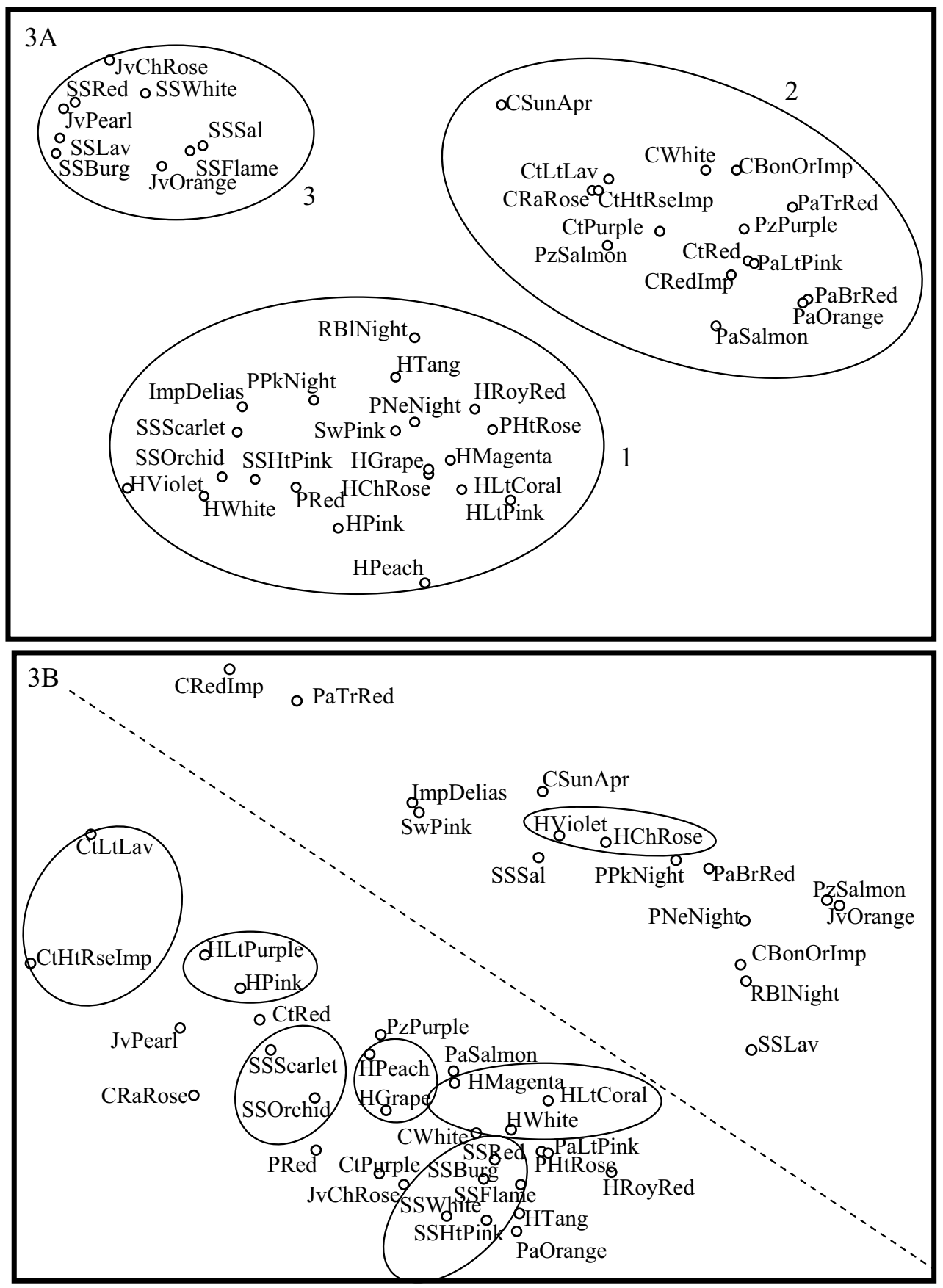

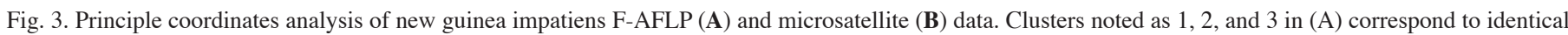
clusters in F-AFLP dendrogram, Fig. 1. Clusters noted in (B) are cultivars within the same series.

analysis provides statistical support for grouping by breeder and series with significant correlations both among cultivars within a breeder $\left(\Phi_{\mathrm{CT}}\right)$ and among series within a breeder $\left(\Phi_{\mathrm{SC}}\right)$. These data suggests that each breeding program uses a distinct set of genetic material for their program overall, and perhaps even for each of their series. The cophenetic correlation coefficients were high for both of the F-AFLP dendrograms, 0.809 and 0.845 for the entire set of cultivars and the partial set of cultivars respectively, indicating a good fit of the tree to the similarity data. Likewise, the principle coordinates supports the same clustering with a significant correlation of 0.758 . This pattern of clustering differs from that of a previous study (Carr et al., 2003); however, the present study encompasses more than twice as many cultivars from five breeders, while the cultivars from the previous study were from a single breeder.

Pedigree information for the cultivars used in this study is limited, making it difficult to compare and verify the results of the marker analyses with known pedigree information. However, studies of other crops such as poinsettia (Parks and Moyer, 2004), wheat (Triticum aestivum L.) (Barrett and Kidwell, 1998), and barley (Schut et al., 1997) have shown that AFLP correlates well with known relationships. Given the limited genetic basis of this hybrid crop and the early dissemination patterns of plant material for cultivation, the clustering may represent the geographical history of these cultivars. In fact, Carr et al. (2003) suggested that groups of new guinea impatiens cultivars that appear to be 
closely related by AFLP analysis may have been derived from species from close geographic regions.

Clustering in the SSR dendrogram was very different from the F-AFLP dendrogram. None of the apparent clustering by breeder was present. However, the cophenetic correlation coefficient for the SSR dendrogram was low, 0.575 , indicating a relatively poor fit of the tree to the similarity data. Conversely, the cophenetic correlation coefficient for the principle coordinates analysis had a higher correlation, at 0.723 , indicating this method graphically displayed similarities between the cultivars more accurately than UPGMA clustering. This diagram (Fig. 3B) shows small clusters of two to five cultivars by series, but still fails to cluster by breeder or series in the same manner as the F-AFLP analysis. AMOVA analysis agrees with the cluster analysis, showing no significant differentiation by breeder or series. To determine the underlying cause of the difference in clustering between the two methods, the similarities were examined more closely. This revealed a pattern to the differences in the F-AFLP and SSR Jaccard similarities. Within-series similarities for the SSR data increased from the F-AFLP Jaccard similarities for every series included in the study. However, the change in clustering patterns was the result of a dramatic increase in similarities between cultivars of different series, which surpassed the small increase in the within series similarities.

Other studies have reported differences in cluster analysis using these markers. A study of olive showed that AFLP would cluster varieties by geographical origin, while microsatellite analysis of the same varieties failed to correlate geographical origin and genetic similarity (Bandelj et al., 2004). Other reports show that while the clustering patterns were comparable using these two methods, AFLP consistently resulted in a higher correlation of pedigree and genetic relationships, including barley (Russell et al., 1997), maize (Zea mays L.) (Pejic et al., 1998), and coconut (Cocos nucifera L.) (Teulat et al., 2000). These differences may occur because AFLP and SSR target different regions of the genome, leading to different measures of similarity (Roy et al., 2004). However, this is confounded with a possible effect from a difference in the number of AFLP fragments and SSR alleles used in this and other comparative studies, due to the nature of these methodologies. In other studies, the difference in the number of polymorphic AFLP fragments and SSR alleles used ranges from 2-fold in barley (Russell et al., 1997) to 17-fold more AFLP fragments in mangrove (Maguire et al., 2002), compared to 3.8-fold more AFLP fragments in the current study. Interestingly, the comparative study of mangrove resulted in a congruence of the taxonomic patterns (Maguire et al., 1997), implying that a difference in the numbers of fragments and alleles may not have an effect on the final cluster analysis.

This study supports the choosing of marker systems for particular applications according to their characteristics as well as practical and economic considerations (Belaj et al., 2003). Both AFLPand SSR have been shown to be effective for differentiating cultivars. However, based on this and other studies, AFLP may be more appropriate for defining relationships than SSR (Maguire et al., 2002; Russell et al., 1997; Teulat et al., 2000). It has been proposed that microsatellites could be useful, for example, to expose "essentially derived" varieties, due to their inability to distinguish between sport mutations and their progenitors (Becher et al., 2000), which could be useful for plant cultivar protection. Heterozygosity values were reported as an indication of the informativeness of a marker system. In this study, these values had a similar range for F-AFLP and SSR, but the average value is higher for F-AFLP, suggesting it may be more informative for fingerprint analysis of new guinea impatiens.

This research advanced the capabilities for genetic analysis of new guinea impatiens. The value of F-AFLP fingerprinting for new guinea impatiens cultivar differentiation was demonstrated and a set of polymorphisms that can be used to identify cultivars was validated, resulting in a database for future cultivar comparisons. Microsatellite loci were identified and tested using semi-automated fluorescent methods. F-AFLP and SSR were shown to be powerful tools for discriminating a diverse set of new guinea impatiens cultivars, with F-AFLP being additionally useful for clustering cultivars by breeder and series. This study validates the robustness of two fingerprinting methods in new guinea impatiens. They may both be useful for purposes of patent validation, cultivar protection, and enhancement of breeding programs.

\section{Literature Cited}

Arisumi, T. and H.M. Cathey. 1976. The new guinea impatiens. HortScience 11:2.

Arisumi, T. 1978. Hybridization among diploid and tetraploid forms of new guinea, java, and celebs Impatiens spp.. J. Amer. Soc. Hort. Sci. 103: 355-361.

Alvarez, A.E., C.C.M. van de Wiel, M.J.M. Smulders, and B.Vosman. 2001. Use of microsatellites to evaluate genetic diversity and species relationships in the genus Lycopersicon. Theor. Appl. Genet. 103:1283-1292.

Bandelj, D., J. Jakse, and B. Javornik. 2004. Assessment of genetic variability of olive varieties by microsatellite and AFLP markers. Euphytica 136:93-102.

Barcaccia, G., E.E. Albertini, and M. Falcinelli. 1999. AFLP fingerprinting in Pelargonium peltatum: its development and potential in cultivar identification. J. Hort. Sci. Biotechnol. 74:243-250.

Barrett, B.A. and K.K. Kidwell. 1998. AFLP-based genetic diversity assessment among wheat cultivars from the Pacific Northwest. Crop Sci. 38:1261-1271.

Becher, S.A., K. Steinmetz, K. Weising, S. Boury, D. Peltier, J.P. Renou, G. Kahl, and K. Wolff. 2000. Microsatellites for cultivar identification in Pelargonium. Theor. Appl. Genet. 101:643-651.

Belaj, A., Z. Satovic, G. Cipriani, L. Ba;doni, R. Testolin, L. Rallo, and I. Trujillo. 2003. Comparitive study of the discriminating capacity of RAPD, AFLP, and SSR markers and of their effectiveness in establishing genetic relationships in olive. Theor Appl. Genet. 107:736-744.

Benham, J.J. 2001. Genographer. 9 Jan. 2006. <http://hordeum.oscs. montana.edu/genographer/>.

Cardle, L., L. Ramsay, D. Milbourne, M. Macaulay, D. Marshall, and R. Waugh. 2000. Computational and experimental characterization of physically clustered simple sequence repeats in plants. Genetics $156: 847-854$

Carr, J., M. Xu, J.W. Dudley, and S.S. Korban. 2003. AFLP analysis of the genetic variability in new guinea impatiens. Theor. Appl. Genet. 106:1509-1516.

Carr, J. and S.S. Korban. 2004. Evaluating genetic relationships in seed impatiens, Impatiens walleriana, using AFLP profiling. Plant Breeding 123:577-581.

Carriero, F., G. Fontanazza, F. Cellini, and G. Giorio. 2002. Identification of simple sequence repeats (SSRs) in olive (Olea europaea L.). Theor. Appl. Genet. 104:301-307.

Debener, T., T. Janakiram, and L. Mattiesch. 2000. Sports and seedlings of rose varieties analysed with molecular markers. Plant Breeding 119:71-74.

De Riek, J., J. Dendauw, M. Mertens, M. De Loose, J. Heursel, and E. Van Blockstaele. 1999. Validation of criteria for the selection of AFLP markers to assess the genetic variation of a breeders' collection of evergreen azaleas. Theor. Appl. Genet. 99:1155-1165.

Ecke, P., Jr. 1998a. The softening of ethics in the breeding industry, Part I. Greenhouse Product News 8:36-37. 
Ecke, P., Jr. 1998b. The softening of ethics in the breeding industry, Part II. Greenhouse Product News 8:16-17.

Esselink, G.D., M.J.M. Smulders, and B. Vosman. 2003. Identification of cut rose (Rosa hybrida) and rootstock varieties using robust sequence tagged microsatellite site markers. Theor. Appl. Genet. 106: 277-286.

Excoffier, L., P.E. Smouse, and J.M. Quattro. 1992. Analysis of molecular variance inferred from metric distances among haplotypes: Application to human mitochondrial DNA restriction data. Genetics 131:479-491.

Han, T.H., H.J. Van Eck, M.J. De Jeu, and E. Jacobsen. 1999. Optimization of AFLP fingerprinting of organisms with a large-sized genome: A study on Alstroemeria spp. Theor. Appl. Genet. 98: 465-471.

Han, T.H., M. De Jeu, H. Van Eck, and E. Jacobsen. 2000. Genetic diversity of Chilean and Brazilian Alstroemeria species assessed by AFLP analysis. Heredity 84: 564-569.

Incirli, A. and M.S. Akkaya. 2001. Assessment of genetic relationships in durum wheat cultivars using AFLP markers. Genet. Resources Crop. Evol. 48:233-238.

Jaccard, P. 1908. Novelles recgerches sur la distribution florale. Bulletin de la Société Vaudoise des sciences naturelles 44:223-270.

Ling, J., R. Sauve, and N. Gawel. 1997. Identification of poinsettia cultivars using RAPD markers. HortScience 32:122-124.

Lyerly, W.J. 2002. Conflag: A Perl script for conversion of ABI Genescan data. 15 Oct. 2003. <https://www.sonicbunny.org/twiki/bin/view/Science/ConFlag $>$.

Maguire, T.L., R. Peakall, and P. Saenger. 2002. Comparative analysis of genetic diversity in the mangrove species Avicennia marina (Forsk.) Vierh. (Avicenniaceae) detected by AFLPs and SSRs. Theor. Appl. Genet. 104:388-398.

Parks, E.J. and J.W. Moyer. 2004. Evaluation of AFLP in poinsettia: Polymorphism selection, analysis, and cultivar identification. J. Amer. Soc. Hort. Sci. 129:863-869.

Pejic, I., P.Ajmone Marsan, M. Morgante, V. Kozumplick, P. Castiglioni, G. Taramino, and M. Motto. 1998. Comparative analysis of genetic similarity among maize inbred lines detected by RFLPs, RAPDs, SSRs, and AFLPs. Theor. Appl. Genet. 97:1248-1255.

Rampling, L.R., N. Harker, M.R. Shariflou, and M.K.Morell. 2001. Detection and analysis systems for microsatellite markers in wheat. Austral. J. Agr. Res. 52:1131-1141.
Roy, J.K., M.S. Lakshmikumaran, H.S. Balyan, and P.K. Gupta. 2004 AFLP-based genetic diversity and its comparison with diversity based on SSR, SAMPL, and phenotypic traits in bread wheat. Biochem. Genet. 42:43-59.

Rozen, S. and H. Skaletsky. 2000. Primer3 on the WWW for general users and for biologist programmers, p. 365-386. In: S. Krawetz and S. Misener (eds.). Bioinformatics methods and protocols: Methods in molecular biology. Humana Press, Totowa, N.J.

Russell, J.R., J.D. Fuller, M. Macaulay, B.G. Hatz, A. Jahoor, W. Powell, and R. Waugh. 1997. Direct comparison of levels of genetic variation among barley accessions detected by RFLPs, AFLPs, SSRs and RAPDs. Theor. Appl. Genet. 95:714-722.

Schut, J.W., X. Qi, and P. Stam. 1997. Association between relationship measures based on AFLP markers, pedigree data and morphological traits in barley. Theor. Appl. Genet. 95:1161-1168.

Smulders, M.J.M., Y. Noordijk, W. Rus-Kortekaas, G.M.M. Bredemeijer, and B. Vosman. 2003. Microsatellite genotyping of carnation varieties. Theor. Appl.Genet. 106:1191-1195.

Temnykh, S., G. DeClerck, A. Lukashova, L. Lipovich, S. Cartinhour, and S. McCouch. 2001. Computational and experimental analysis of microsatellites in rice (Oryza sativa L.): frequency, length variation, transposon associations, and genetic marker potential. Genome Res. 11:1441-52.

Teulat, B., C. Aldam, R. Trehin, P. Lebrun, J.H.A. Barker, G.M. Arnold, A. Karp, L. Baudouin, and F. Rognon. 2000. An analysis of genetic diversity in coconut (Cocos nucifera) populations from across the geographic range using sequence-tagged microsatellites (SSRs) and AFLPs. Theor. Appl. Genet. 100:764-771.

Tomkins, J.P., T.C. Wood, L.S. Barnes, A. Westman, and R.A. Wing. 2001. Evaluation of genetic variation in the daylily (Hemerocallis spp.) using AFLP markers. Theor. Appl. Genet. 102:489-496.

Vos, P., R. Hogers, M. Bleeker, M. Reijans, T. van de Lee, M. Hornes, A. Frijters, J. Pot, J. Peleman, M. Kuiper, and M. Zabeau. 1995. AFLP: A new technique for DNA fingerprinting. Nucleic Acids Res. 23:4407-4414.

Weddington, M. 2002. Halting plant identity theft. FloraCulture Intl. $12: 44-47$.

Xiang, N. and Y. Hong. 2003. Genetic analysis of tropical orchid hybrids (Dendrobium) with fluorescence amplified fragment-length polymorphism (AFLP). J. Amer. Soc. Hort. Sci. 128:731-735. 